\title{
Desenvolvimento de um sensor visual para o monitoramento de temperatura
}

\author{
A. V. SOUZA ${ }^{1}$, M.E.BENEDET ${ }^{2}$, R.WESTPHAL ${ }^{1}$, I. MERGEN ${ }^{1}$ e R.A.F.MACHADO ${ }^{1}$ \\ ${ }^{1}$ Universidade Federal de Santa Catarina, Departamento de Engenharia Química e Engenharia de \\ Alimentos \\ ${ }^{2}$ Universidade Federal de Santa Catarina, Departamento de Engenharia \\ Mecânica \\ E-mail para contato: aline.v@posgrad.br
}

\begin{abstract}
RESUMO - Os materiais ditos inteligentes são materiais funcionais projetados para servir a propósitos específicos de uma forma controlada, como para atuação em ambientes especiais ou de difícil acesso ou mesmo para que apresente uma resposta a um dado evento. Entre os materiais funcionais, em especifico, a classe dos compostos termocrômicos permite o desenvolvimento de aplicações para o monitoramento visual da temperatura. Neste trabalho foi utilizado 1,3,3-Trimetilidolino-beta-naftoespiropiran (comercialmente denominada de Spyropiran T0423) para o desenvolvimento de um sensor aplicado ao monitoramento de temperatura em diversos tipos de processos. O Spyropiran foi aplicado em um substrato contendo um verniz poliuretânico e as mudanças de temperaturas foram monitoradas observando as alterações de cor para caracterização deste sensor. Ensaios para determinação das condições de aplicação, como a análise de ciclo de resistência térmica e aderência ao aço inox, também foram realizados.
\end{abstract}

\section{INTRODUÇÃO}

O spiropyran(SP) é um tipo de composto químico orgânico conhecido por possuir propriedades foto, mecano e termocrômicas. O SP quando é submetido ao aquecimento (termocromismo) ou radiação (fotocromismo) torna-se colorido. A estrutura química do SP possui uma ligação de carbono com oxigênio fraca, a qual se torna de fácil rompimento sob irradiação UV ou temperatura ou forças mecânicas. A figura abaixo mostra uma molécula SP que quando aquecida ou por radiação UV promove um rompimento do anel (CO) para uma estrutura aberta chamada merocianina (MC).

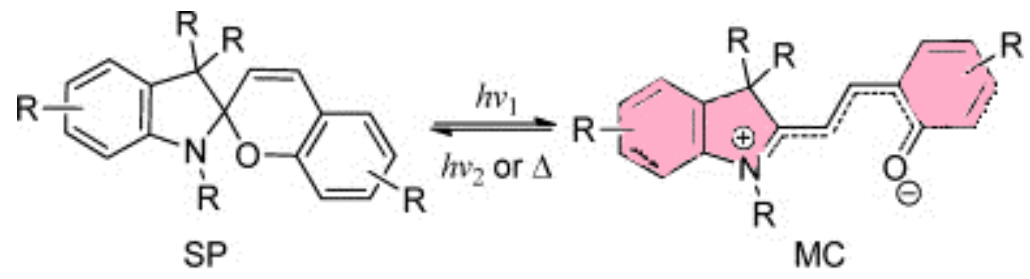


Figura 1 - Estruturas químicas do spiropiran (SP) e sua forma aberta merocianina (MC). Transformação do SP.

Essa diferença de cor pode ser diferente para diferentes estruturas moleculares de spiropyran. Neste trabalho, o composto utilizado foi aplicado em uma base de Poliuretano acrílico que quando submetido a um determinado aquecimento altera sua cor e tonalidade. Na tabela 1, pode-se observar as especificações do pigmento analisado.

Tabela 1 - Moléculas SP e suas características.

\begin{tabular}{|c|c|c|c|c|}
\hline Código & $\begin{array}{c}\text { Forma } \\
\text { Molecular }\end{array}$ & Nome & Molécula & Característica \\
\hline $\mathrm{T} 0423$ & $\mathrm{C}_{23} \mathrm{H}_{21} \mathrm{NO}$ & $\begin{array}{c}1,3,3- \\
\text { Trimethylindoli } \\
\text { no-beta- } \\
\text { naphthopyrylos } \\
\text { piran }\end{array}$ & \begin{tabular}{c} 
Termocrômico \\
\hline
\end{tabular}
\end{tabular}

\subsection{Termocromismo}

O termocromismo pode ser induzido de forma direta ou indireta. No termocromismo direto, o fenômeno ocorre por influência da temperatura sobre o composto no qual a variação espectral ocorre. No termocromismo indireto, a temperatura influi sobre o meio no qual o composto termocrômico está inserido, provocando a resposta termocrômica. Esse fenômeno pode ocorrer em compostos inorgânicos por causas como transições de fase, spin crossover induzido, diminuição do band gap em sólidos condutores, variações na esfera de coordenação e reações químicas reversíveis (El-ayaan et al., 2001; Bamfield, 2001).

Já nos compostos orgânicos o termocromismo pode ocorrer, dentre outras possibilidades, devido a reações químicas reversíveis, rearranjos moleculares ou mudanças conformacionais.

O termocromismo apresenta-se especialmente interessante devido às suas aplicações científicas, tecnológicas e comerciais em geral. As aplicações dos compostos termocrômicos são várias, desde a confecção de brindes e brinquedos até o acompanhamento de mudanças metabólicas em doenças como o câncer de mama (White et al.,1999).

Algumas aplicações tecnológicas são na área de segurança. Nestes casos, o efeito da cor pode ser aplicado em lugares em que se trabalha com temperaturas extremas, como em laboratórios, indústrias ou oficinas, incluindo a sinalização de máquinas ou motores. Desta forma, o aparecimento de uma cor diferente em um objeto pode alertar sobre a sua temperatura, como em um cadinho ou uma vidraria de laboratório, ou mesmo em uma tampa de radiador de automóvel, evitando assim acidentes com queimaduras.

Na indústria de petróleo e gás pode ser empregado como sensor de temperatura em locais onde é difícil a obtenção de uma medida direta ou mesmo existe a impossibilidade de se instalar um sensor 


\section{9 a 22 de outubro de 2014 \\ Florianópolis/SC}

intrusivo, como por exemplo em colunas de filme descendente.

\subsection{Tinta}

A tinta pode ser considerada como uma mistura estável de uma parte sólida em um componente volátil (água ou solventes orgânicos). Para conferir propriedades específicas são empregados aditivos, os quais são responsáveis pela obtenção de propriedades importantes tanto nas tintas quanto no revestimento. A junção destes itens (sólidos e voláteis) define as propriedades de resistência e de aspecto, bem como o tipo de aplicação e o custo final (Abrafati,2006).

De acordo com o Catálogo de Pintura Moveleira da Farben a tinta é um revestimento, geralmente orgânico, viscoso, constituído por um ou mais pigmentos, dispersos em resina, que ao sofrer o processo de secagem, forma uma película aderente ao substrato conferindo beleza e proteção (Farben Tintas, 2012). Quando essa tinta não contém pigmentos, ela é chamada de verniz. Por ter pigmentos a tinta cobre o substrato, enquanto o verniz o deixa transparente. Para cada finalidade, há um tipo especifico de tinta, tais como:

- Tintas imobiliárias: Destinadas à construção civil, subdivididas em: Produtos Aquosos (látex) e Produtos à base de solventes orgânicos (esmaltes sintéticos, etc);

- Tintas industriais do tipo OEM (original equipament manufacturer): em sua elaboração incluem os fundos (primers) eletroforéticos, primers à base solvente, tintas em pó, tintas de cura por radiação (UV), entre outras;

- Tintas especiais: Nesta categoria encontram-se as tintas do tipo de pintura e repintura automotiva, de demarcação de tráfego, tintas marítimas, moveleira, etc.

\subsection{O problema do monitoramento de temperatura de forma indireta}

Um dos problemas de monitoramento de temperatura é a aplicação indireta em redes de distribuição de energia elétrica nos pontos de derivação, que são sujeitos à formação de pontos de corrosão e por conseguinte, problemas como concentração diferencial, diferença de potencial, acúmulo de pó, entre outros. As redes de distribuição elétrica realizam essa inspeção pelo método de termovisão, onde o monitoramento é feito através de uma pistola de infravermelho nos pontos de aquecimento. Seria vantajosa uma indicação visual sem a necessidade de equipamentos especiais, permitindo um monitoramento de forma direta, onde até mesmo a população local pudesse observar.

Outro exemplo seria na indústria de petróleo e gás, pois não se sabe as condições de temperatura que determinado fluido está dentro de uma tubulação que está submersa à 5000 ou 6000 metros de profundidade, pois não existe sensor de temperatura nesses dutos. Também nesse caso, seria vantajoso um material que indicasse a temperatura, permitindo a inspeção por câmeras.

Além desses, existem diversos outros exemplos onde o sensor visual para monitoramento de temperatura poderia ser utilizado. Assim, tal tecnologia possui aplicação em diversos setores. 


\section{9 a 22 de outubro de 2014 \\ Florianópolis/SC}

\section{METODOLOGIA}

A molécula SP utilizada neste trabalho foi adquirida na TCI Chemical. Além da molécula, foram utilizados: base de poliuretano acrílico(PU), concentrado branco (dióxido de titânio), thinner (solução com solventes aromáticos) e catalisador (endurecedor para PU acrílico) fornecidos pela empresa Farben Tintas.

Para o verniz, adicionou-se 0,8\% em massa de SP, thinner (15\%) para ter uma melhor aplicação e catalisador na proporção de 2:1 em relação à quantidade inicial de base. Aplicou-se com uma pistola de sucção uma fina camada de verniz em uma chapa de aço inox $(7 \times 13 \mathrm{~cm})$.

Para tinta foi escolhida uma base PU acrílico branco, com a composição de $66 \%$ de PU acrílico e $34 \%$ de concentrado branco. Onde foram catalisados na proporção de 2:1 e colocado tinner (15\%).

Para uma melhor visualização da cor, foram realizados testes com uma base PU acrílico branco puro que foi aplicada na peça de aço inox. A peça foi mantida 15 minutos na temperatura ambiente, seguidos de 15 minutos em estufa a $60^{\circ} \mathrm{C}$. Em seguida, a peça foi retirada e aguardou-se mais 10 minutos para resfriar à temperatura ambiente. Posteriormente aplicou-se o verniz sobre esta camada de tinta.

As tintas com o cromóforo SP foram submetidas às análises de Termogravimétrica(TGA) e DSC (Calorimetria Diferencial de Varredura). Foram avaliados os parâmetros de aderência ao aço inox (norma da ABNT NBR 11003), viscosidade (ABNT NBR 5849) e densidade apropriada para aplicação além da análise de intemperismo (resistência à radicação UV-A e condensação de umidade) conforme a norma ASTM G154 e análise de ciclo de resistência térmica.

Para análise de ciclo de resistência térmica, as peças foram aquecidas em uma chapa de aquecimento até $120^{\circ} \mathrm{C}$ com uma taxa de aquecimento de $48,2^{\circ} \mathrm{C} / \mathrm{min}$ e uma taxa de resfriamento de $80^{\circ} \mathrm{C} / \mathrm{min}$, observando-se a mudança de cor com o aumento da temperatura. As imagens foram obtidas por uma câmera Nikon P510.

As análises de Intemperismo foram realizadas na câmara exposta à luz UV-A proveniente de lâmpadas fluorescentes e umidade saturada. Onde $4 \mathrm{~h}$ de exposição à luz UV-A a $65^{\circ} \mathrm{C}+4 \mathrm{~h}$ de exposição à umidade saturada a $50^{\circ} \mathrm{C}$, repetindo este processo finalizando com $720 \mathrm{~h}$.

\section{RESULTADOS E DISCUSSÕES}

A abertura de anel pela ligação química fraca CO produz uma alteração na absorção da luz incidente, o que visualmente é observado através de uma mudança de cor do material. Foram explorados os efeitos visuais a quente usando a chapa de aquecimento, atingindo temperaturas de até $120{ }^{\circ} \mathrm{C}$ monitoradas por uma termocâmera (FLIR T400).

\subsection{Efeito Termocrômico - T0423}

Nesse sensor desenvolvido foi observado o efeito termocrômico. As mudanças de cor foram de branco para um lilás, mudando de cor em torno de $95^{\circ} \mathrm{C}$ (ponto de viragem) e com resistência até $180^{\circ} \mathrm{C}$. 
Esta coloração ocorre devido à abertura da ligação CO pela temperatura, induzindo o movimento do cromóforo e da possível cisão da ligação química fraca.

Na figura 2 é possível observar os resultados da análise de ciclo realizadas com aquecimentos e resfriamentos das peças para inferir sobre o seu tempo de vida. Foram realizados 108 ciclos nos quais a peça foi perdendo o efeito termocrômico e não alterando mais sua cor. A foto(a) apresenta a tinta sendo submetida ao aquecimento nas primeiras vezes e a foto (b) apresenta a centésima oitava vez que foi aquecida onde perdeu completamente sua propriedade termocrômico e acabou ficando um pouco amarelada a uma temperatura de $120^{\circ} \mathrm{C}$. A mudança de cor se deve à mudança química pelo rompimento da ligação química $\mathrm{CO}$ pela perturbação térmica. Este fenômeno termocrômico a $95^{\circ} \mathrm{C}$ demonstrou que o efeito foi reversível no período de resfriamento das amostras. Porém depois de um certo tempo o pigmento acaba perdendo suas propriedades termocrômicas. Entretanto as condições empregadas no presente trabalho são extremas e não corriqueiras na indústria de petróleo e gás.

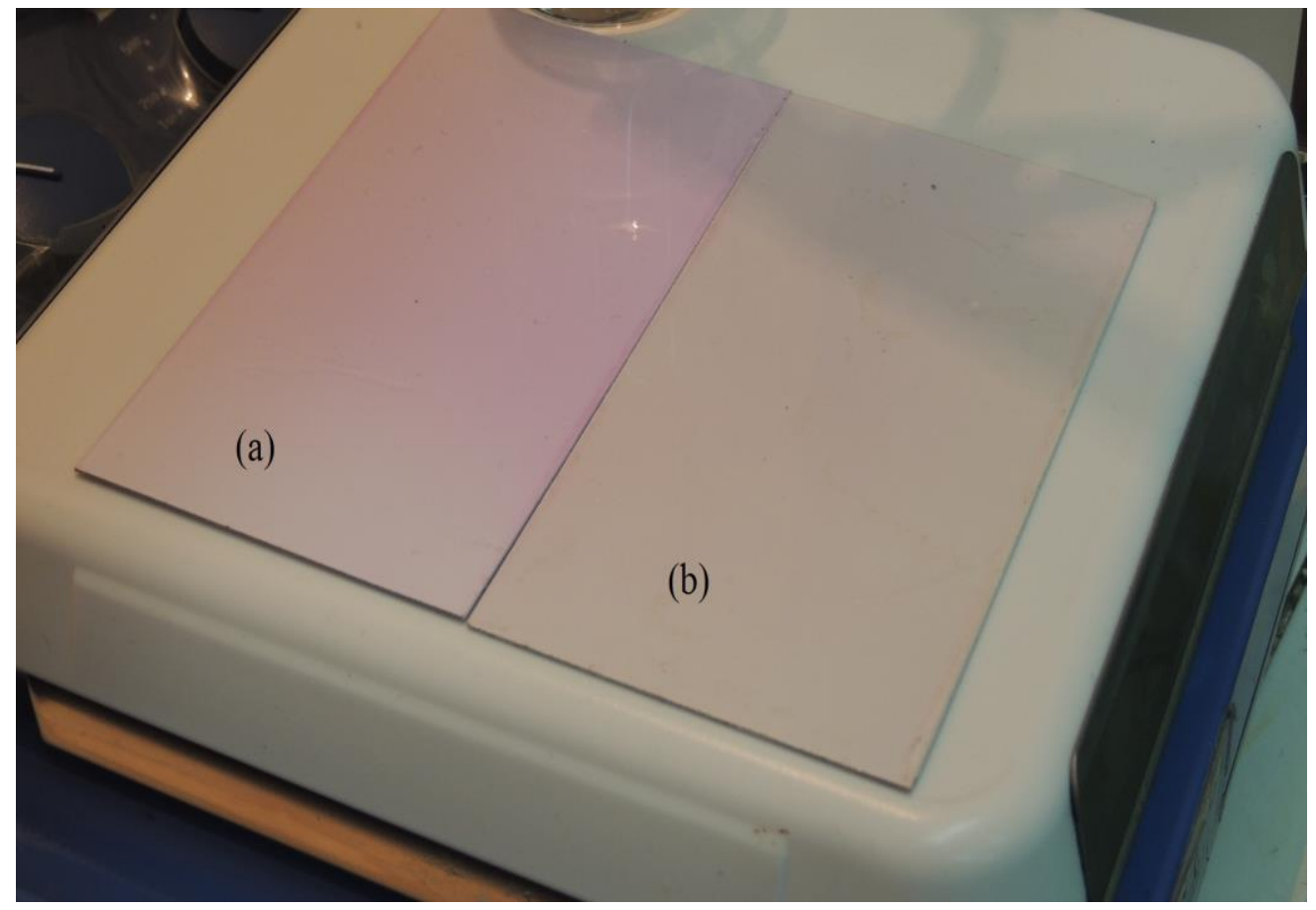

Figura 2 -Foto da análise de ciclo de resistência térmica

Conforme pode ser observado na Figura 3 que apresenta a análise de DSC (Calorimetria Diferencial de Varredura), o pigmento funde em $180^{\circ} \mathrm{C}$ e pela Figura 4 que apresenta a análise de TGA o mesmo começa a degradar em torno dos $200^{\circ} \mathrm{C}$. 


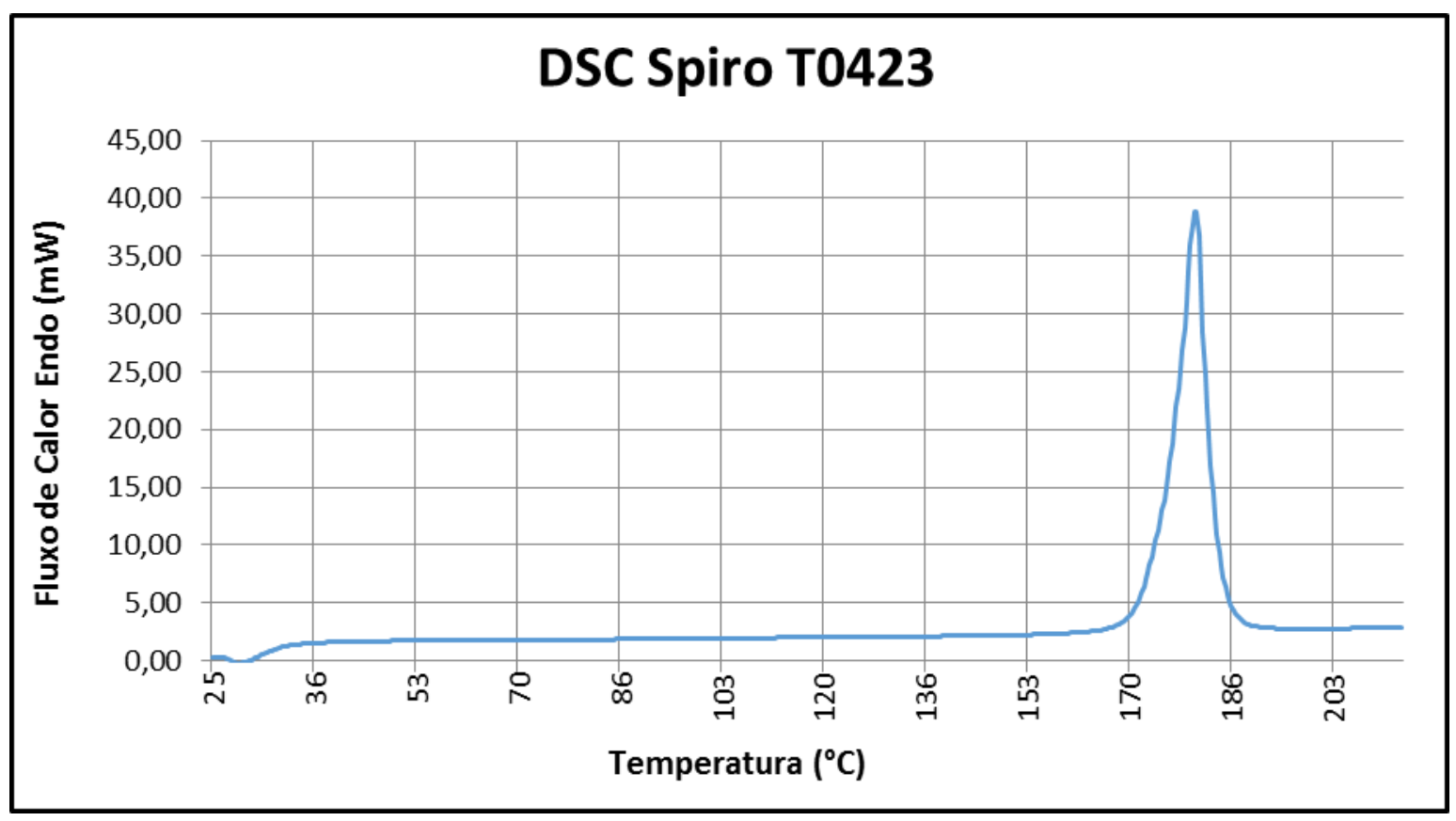

Figura 3 - Análise DSC do SP (T0423)

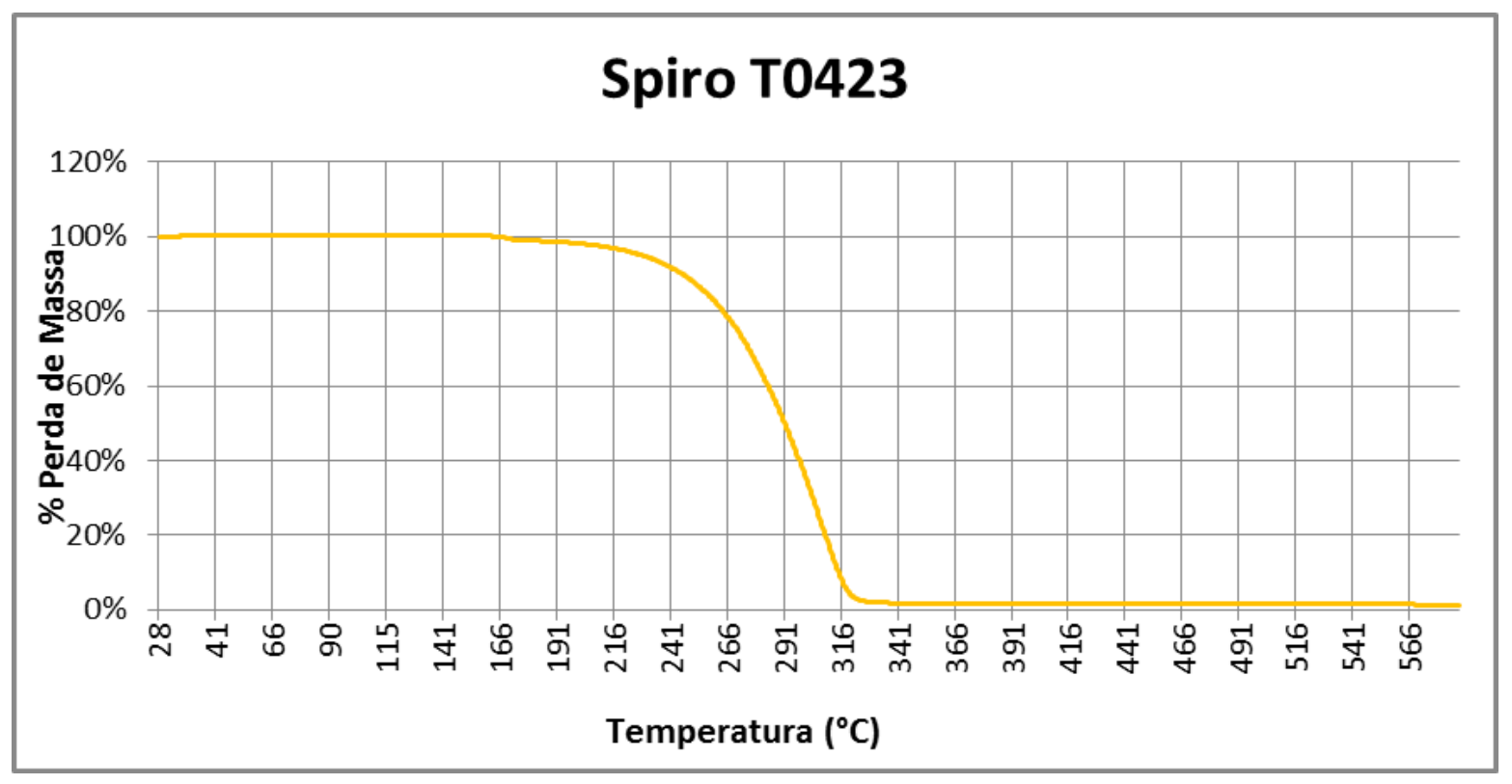

Figura 4 - Análise TGA do SP (T0423)

Foram feitos teste de aderência conforme a Figura 5, pelo método B (corte em grade), onde foi constatado $100 \%$ de aderência da tinta na chapa de aço inox. Para uma melhor aplicação também foi determinado uma densidade de $1,06 \mathrm{~g} / \mathrm{cm}^{3}$ e viscosidade de $18 \mathrm{cps}$ (Copo Ford $\mathrm{n}^{0}{ }^{4}$ ) o qual apresentou uma excelente condição ou aplicação. 


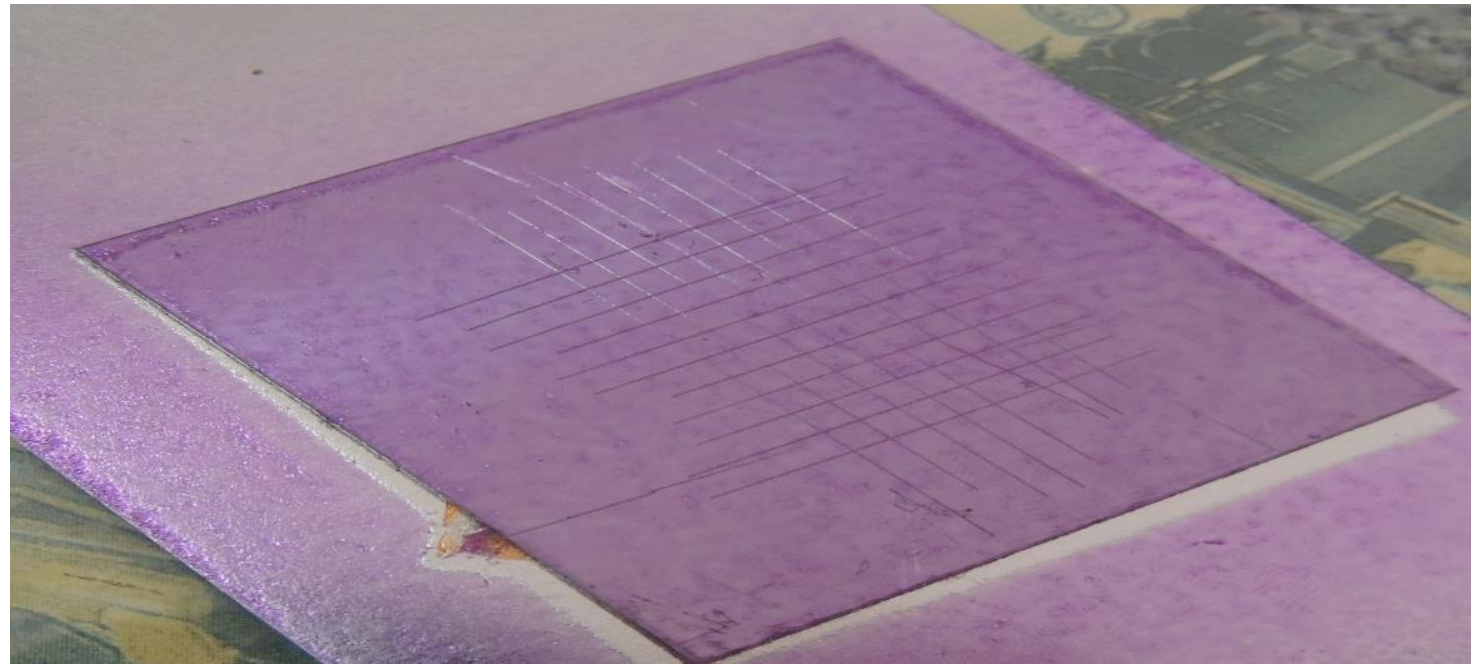

Figura 5 - Teste de Aderência da Tinta com o SP.

A análise de intemperismo abrange os princípios básicos e procedimentos operacionais para a utilização de luz UV fluorescente e aparelho de água destinada a reproduzir os efeitos de intemperismo que ocorrem quando os materiais são expostos à luz solar (direta ou através do vidro da janela) e umidade em forma de chuva ou orvalho em uso real. Esta prática é limitada aos procedimentos para a obtenção, medição e controle das condições de exposição.

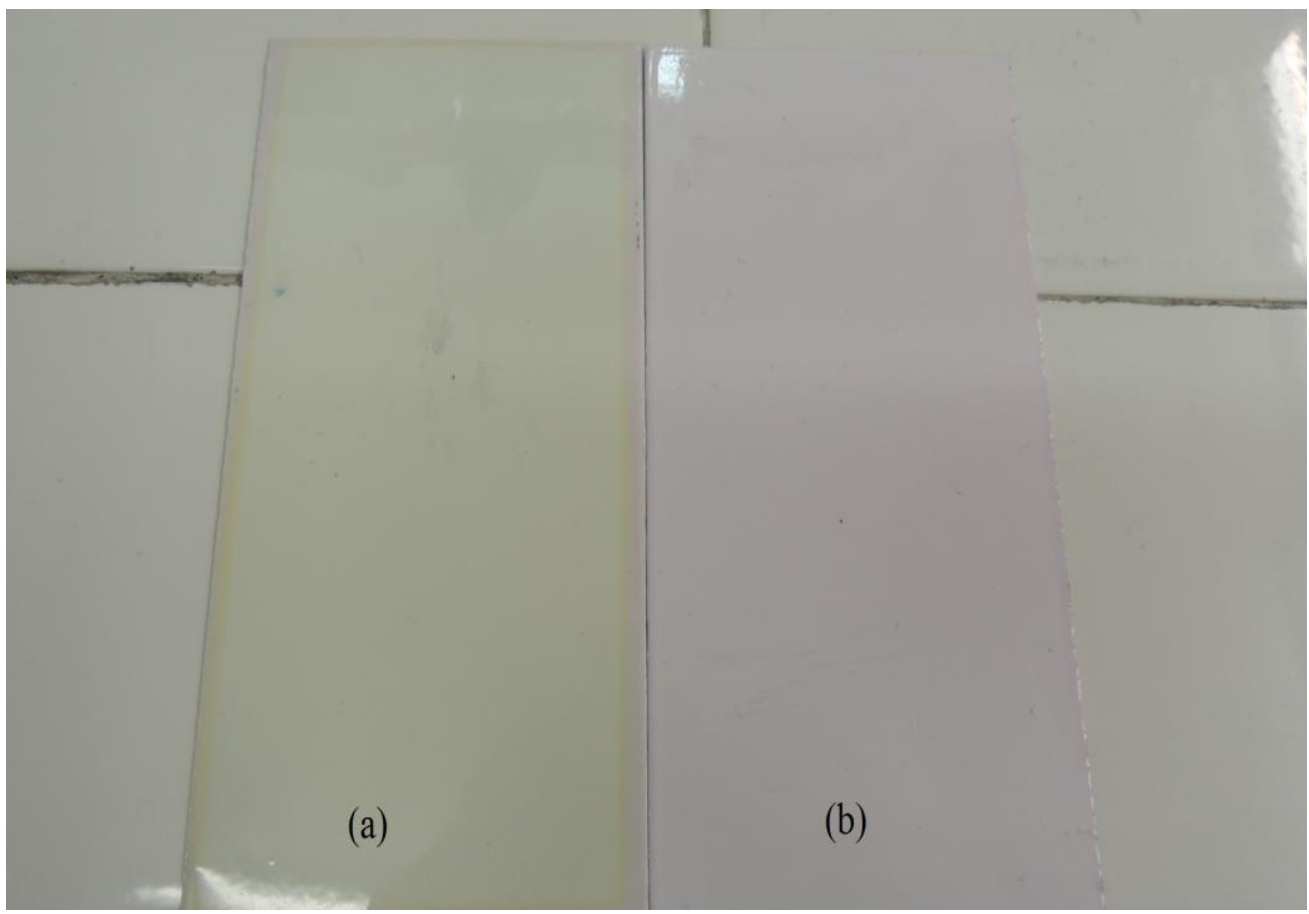

Figura 6: Análise de Intemperismo 
Como podemos notar na Figura 6, a foto(a) foi exposta na câmara por $720 \mathrm{~h}$ e ficou com uma cor amarelada e desbotada, onde se perdeu toda sua cor original comparada com a foto (b). Isso pode estar acontecendo pelo fato do SP estar reagindo com a luz UV ou pelo fato de não suportar tanto tempo na câmara. Tal fenômeno será mais criteriosamente estudado para determinar as mudanças a serem realizadas na composição da tinta.

\section{CONCLUSÕES}

O desenvolvimento do sensor tem como objetivo a aplicação em equipamentos que trabalhem nessa faixa de temperaturas, entre $\left(95\right.$ a 170) ${ }^{\circ} \mathrm{C}$, pois com o acréscimo da temperatura ele vai intensificando sua cor ficando cada vez mais lilás, possuindo uma infinidade de aplicações na área industrial. Este sensor mostrou-se com uma excelência propriedade termocrômica com apenas $0,8 \%$ na sua fórmula, tendo resultados satisfatórios.

\section{REFERENCIAS}

ABRAFATI. Guia Técnico Ambiental Tintas e Vernizes - Série P+L. Federação das Indústrias do Estado de São Paulo - FIESP. 2006. 70 p.

BAMFIELD, P. Chromic Phenomena - The Technological Applications of Colour Chemistry - The Royal Society of Chemistry, 2001.

EL-AYAAN, U; MURATA, F.; FUKUDA, Y. Thermochromism and Solvatochromism in Solution Monatshefte für Chemie Chemical Monthly, Cidade v. 132, n.11, p,1279-1294, 2001.

FARBEN TINTAS. Curso de Pintura Moveleira. 2012. 27 p.

WHITE, M. A; LEBLANC, M. Thermochromism in Commercial Products. Journal of Chemical Education, v. 76, n.9, p. 1201-1205, 1999.

Disponível em: < http://www.abntcatalogo.com.br/norma.aspx?ID=3239>Acesso em: 20 mar. 2014.

Disponível em:

ftp://ftp.demec.ufpr.br/disciplinas/TM734/AT\%20P\%F3s\%202014/NBR11003\%20Determina\%E7\%E3o \%20ader\%EAncia.pdf> Acesso em: 22 mar. 2014.

Disponível em: < http://dc184.4shared.com/doc/yp1_vlzW/preview.html> Acesso em: 23 mar. 2014. 